\title{
Consideraciones en el manejo clínico durante procedimientos odontológicos conservadores
}

Taking some considerations about minimally invasive dentistry procedures during clinical managament

Juan Portocarrero ${ }^{1}$

\section{Sr. Editor:}

Resulta importante evidenciar a través de la práctica clínica diaria, el uso de los diferentes materiales odontológicos que surgen actualmente, con el objetivo de brindar funcionalidad y estética a los pacientes, teniendo en cuenta cuán importante es la preservación de una pieza dentaria para el correcto funcionamiento del Sistema Estomatognático, como se muestra en el reporte de caso publicado por Yfuma Pedroza J. y col (1), en el último volumen de la prestigiosa revista que dignamente dirige, el cuál aborda el tema de protección pulpar mediante el uso de cementos de silicato.

Considero que el reporte de caso presentado es importante para su difusión y felicito a los autores por demostrar que la odontología debe basarse en evidencia científica, además por dar a conocer cuánto hay por seguir investigando sobre el tema abordado. Sobre el reporte de caso, permítame expresar algunas consideraciones que puedan contribuir para posteriores situaciones clínicas:
Actualmente el diagnóstico sistematizado de la caries dental es fundamental para iniciar una odontología de mínima intervención (2), lo cual implica que la lesión cariosa debe ser diagnosticada mediante un sistema íntegro que contemple su valoración, inicio y progresión de la misma, como lo hace, actualmente, el Sistema Internacional de Detección y Valoración de la Caries dental I y II (ICDAS por sus siglas en inglés), permitiéndonos evidenciar la lesión en función de su compromiso y su futuro tratamiento (3), lo cual considero que debió indicarse en el reporte de caso.

Considero también que la consignación de un examen auxiliar, debió ser necesario. El informe imagenológico de una radiografía aleta de mordida, era pertinente para determinar la probable profundidad y compromiso del complejo dentino pulpar, $(4,5)$ y valorar la remoción parcial o total de dentina con tejido deficiente, para posteriormente seleccionar con certeza, el sistema adhesivo a utilizar. $\mathrm{Si}$ bien es cierto el tema tratado en el presente reporte 
de caso, está únicamente dirigido al uso del material odontológico a base de silicato de calcio, como protector pulpar, pero debemos hacer hincapié en las razones de la elección del sistema adhesivo adecuado según el diagnóstico de la lesión cariosa, y que según la evidencia científica disponible, el uso de sistemas adhesivos auto-acondicionante de dos pasos es viable si las lesión cariosa está ubicada en dentina profunda, proporcionando mayor fuerza de adhesión en dentina en relación a los adhesivos auto-grabantes de un solo paso que proporcionan menor fuerza de unión en el sustrato dentinario (6).

Por otro lado al mencionar en el reporte de caso, la inexistencia de protocolos ideales para preservar la vitalidad pulpar, podría causar cierta confusión entre la comunidad científica, ya que los procedimientos de recubrimiento pulpares (RPI y RPD por sus siglas en español) son considerados como procedimientos conservadores, con el objetivo final de salvaguardar y mantener la vitalidad pulpar de las piezas afectadas (7), con indicaciones previas como por ejemplo: Condición sistémica del paciente, la edad de la pieza dentaria, la técnica de remoción cariosa utilizada, los signos clínicos de vitalidad pulpar, y consideraciones imagenológicas que evidencien la conservación normal de los tejidos a nivel óseo y periapical. Todo lo anteriormente descrito debe estar basado en el diagnóstico sistematizado de la lesión cariosa (7).

Finalmente, es importante mencionar que el reporte de caso publicado resulta muy beneficioso para los odontólogos clínicos, pues permite el debate y hace de la odontología una ciencia basada en la mejor evidencia científica disponible, en aras de ser utilizada en el abordaje clínico hacia los pacientes.

\section{Correspondencia}

Juan Pablo Portocarrero Mondragón.

Km 4 a Carretera Pimentel

Pimente, Chiclayo, Perú

Correo electrónico: pmondragonju@crece.uss.edu.pe

\section{REFERENCIAS BIBLIOGRÁFICAS}

1. Yfuma J, Ñaupari R, Noborikawa A, Nuñez M. Protección pulpar: cementos a base de silicato de calcio. Relato de un caso clínico. Rev Estomatol Herediana. 2020;30(3):196-200. DOI: 10.20453/reh. v30i3.3823

2. Giacaman RA, Muñoz-Sandoval C, Neuhaus KW, Fontana M, Chałas R. Evidence-based strategies for the minimally invasive treatment of carious lesions: Review of the literature. Adv Clin Exp Med. 2018; 27(7):10091016. doi: $10.17219 / \mathrm{acem} / 77022$

3. Taqi M, Razak IA, Ab-Murat N. Comparing dental caries status using Modified International Caries Detection and Assessment System (ICDAS) and World Health Organization (WHO) indices among school children of Bhakkar, Pakistan. J Pak Med Assoc. 2019; 69(7):950-954. (Citado el 18/11/2020) Disponible en: https://jpma.org.pk/article-details/9226?article_ id $=9226$

4. Goodwin TL, Devlin H, Glenny AM, O'Malley L, Horner K. Guidelines on the timing and frequency of bitewing radiography: a systematic review. Br Dent J. 2017; 222(7):519-526. Doi: 10.1038/sj.bdj.2017.314

5. Schwendicke F, Tzschoppe M, Paris S. Radiographic caries detection: A systematic review and metaanalysis. J Dent. 2015; 43: 924-933. doi:10.1016/j. jdent.2015.02.009

6. Bin Hasan M. Self-etching Adhesive Systems in Operative Dentistry: A Literature Review. Research \& Reviews: Journal of Dental Sciences. 2017; 5(2):2332. (Citado el 21/11/2020/ Disponible en: https://www. rroij.com/open-access/selfetching-adhesive-systemsin-operative-dentistry-a-literature-review-. php?aid $=85984$

7. Miyashita H, Worthington HV, Qualtrough A, Plasschaert A. Pulp management for caries in adults: maintaining pulp vitality. Cochrane Database Syst Rev. 2007; 2: CD004484. DOI: 10.1002/14651858. CD004484.pub2 